LII Zakopane School of Physics, International Symposium Breaking Frontiers, Zakopane, Poland, May 22-27, 2017

\title{
On the Possibility to Control an Atom Motion in a FCC Iron Nanocluster
}

\begin{abstract}
N.V. Bondarenko* ANd A.V. Nedolya
Applied Physics and Nanomaterials Department, Zaporizhzhya National University, Zaporizhzhya, Ukraine

The energy of the isolated iron nanocluster was calculated by molecular mechanics method using the LennardJones potential depending on the position of impurity carbon atom and substitutional atoms of nickel. The cluster included a carbon atom, that drifted from an inside octahedral interstice to a direction $\langle 022\rangle$ to the surface directly or to a tetrahedral interstice in $\langle\overline{1} 11\rangle$ direction and after that in $\langle 222\rangle$ direction to the surface. In addition one of 14 iron atoms was replaced by a nickel atom (or pair atoms), the position of which was changing during simulation. It is shown that there were positions of a nickel atom that significantly affected nanoclusters energy. The calculation results indicated that position of a carbon atom in the octahedral interstice was more energetically favorable than tetrahedral interstice in the case of fcc nanocluster. On the other side, the potential barrier was smaller in the direction $\langle\overline{1} 11\rangle$ than in the direction $\langle 022\rangle$. This indicates that there are two ways for carbon atom to drift to the surface of the nanocluster. The positions of nickel atoms were identified, which significantly affected the height of potential barriers of a tetrahedral and an octahedral interstice and determined the possible direction of carbon atoms drift. This allows manipulating atoms at the surface of nanocluster.
\end{abstract}

DOI: 10.12693/APhysPolA.133.277

PACS/topics: 02.70.Ns, 07.05.Pt , 36.40.-c, 61.46.Hk, 61.46.Bc

\section{Introduction}

Usually, the nanostructured materials and nanoparticles are created from traditional metal alloys under the influence of the extreme conditions: extrusion, multiple phase transitions, laser surface treatment, metal particles deposition from the vapor phase, etc. $[1,2]$. As a result, the metastable phases can be obtained because of the high cooling rate, high degrees of deformation or both $[3,4]$.

In any case, the obtained nanostructures are quasistable and change their properties over time $[5,6]$.

The study of the metastable nanostructures and nanoclusters can help solve the problem of their stabilization using the atoms of other types. Also, it is possible to control the properties of nanoparticles by changing the spatial configuration of other type atoms.

\section{Model}

For the study, we chose an fcc $\mathrm{Fe}-\mathrm{Ni}-\mathrm{C}$ nanocluster containing 15 atoms. We assumed that such a cluster forms randomly at initial time and contains one carbon atom and one nickel atom, that substitutes iron atom. The system was considered to be quasi-stable and quasiisolated, that is why it was only statics that we took into account when estimating energy changes using molecular mechanic method $(\mathrm{MM}+$ algorithm). We chose the fcc cluster because all the atoms in it are located on the surface or formed the surface, which simplified interpretation of calculation results.

We performed an evaluation of energy empirically using the solution of the Newton system of equations

\footnotetext{
*corresponding author; e-mail: nat.v.bondarenko@gmail.com
}

$$
m_{i} \frac{\mathrm{d}^{2} r_{i}(t)}{\mathrm{d} t^{2}}=-\frac{\partial U\left(r_{i, j}\right)}{\partial r_{i}}+\boldsymbol{F}_{i}
$$

where the Lennard-Jones (LJ) pair-potential was $U\left(r_{i j}\right)=4 \varepsilon_{k l} \sum_{i<j}\left[\left(\frac{\sigma_{k l}}{r_{i j}}\right)^{12}-\left(\frac{\sigma_{k l}}{r_{i j}}\right)^{6}\right]$ and where $\varepsilon_{k l}=$ $\sqrt{\varepsilon_{k k} \varepsilon_{l l}}$ - the bond energy and $\sigma_{k l}=\frac{\sigma_{k k}+\sigma_{l l}}{2}$ - the measure of the atomic size, were calculated using the Lorenz-Berthelot mixing rule of atoms of $k$-th and $l$-th classes; $\boldsymbol{F}_{i}$ - the force that determines intermolecular interactions; $r_{i}$ and $r_{j}$ - the coordinates of the interacting atoms $r_{i j}=\left|\boldsymbol{r}_{i}-\boldsymbol{r}_{j}\right|$ [7-9].

The choice of LJ potential was associated with the fact that the size of nanocluster was less than a critical size (less than $1 \mathrm{~nm}$ ) and the random forming of fcc of similar structure did not mean that it was crystalline in every sense of the word, because it was smaller than three coordination spheres of atoms. Thus, for simplicity of calculations for the nanocluster, we preferred the generalized Lennard-Jones potential over other potentials (the Buckingham potential or truncated L-J potential).

Due to the fact that the energy in such calculations is determined up to a constant, we calculated the energy difference between the position of atom of carbon inside of the nanocluster in the octahedral or the tetrahedral interstices and the current position during its drift to the surface

$$
\Delta u=u(L)-u(0),
$$

where $L$ is a length of the carbon atom path, $u$ - specific potential energy. The position of the carbon atom in the central octahedral interstitial site (COIS) of a cluster was chosen as null (0) of the path length $(L)$, conforming to the central symmetry of the nanocluster. We also numbered their positions for convenience (see Fig. 1).

We considered the movement of a carbon atom as similar to the drift to surface due to the influence of surface 


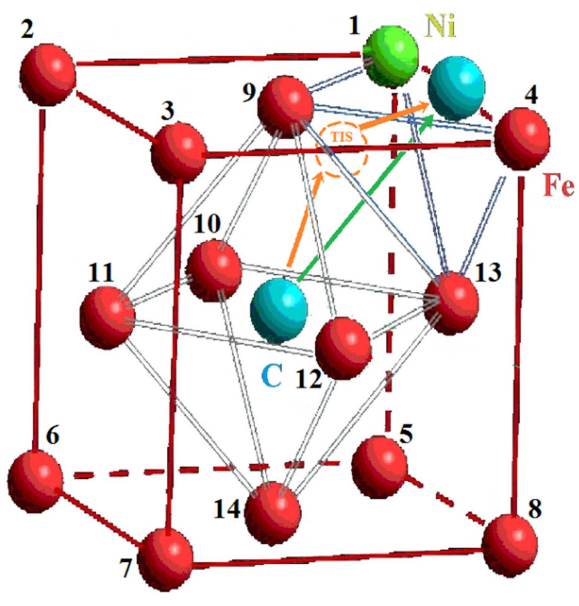

Fig. 1. The scheme of carbon atom drift to the surface: green arrow - in the $\langle 022\rangle$ direction; orange arrows in the $\langle\overline{1} 11\rangle$ plus $\langle 222\rangle$ directions.

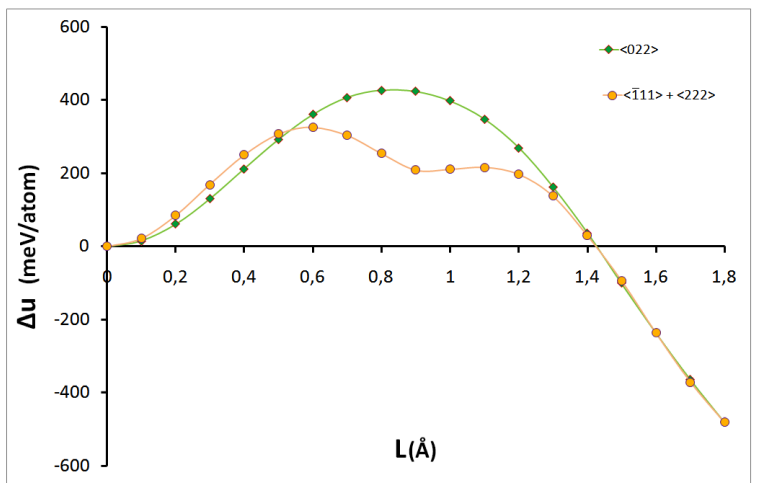

Fig. 2. The specific energy change of the iron nanocluster during the carbon atom drift in the $\langle 022\rangle$ and $\langle\overline{1} 11\rangle$ plus $\langle 222\rangle$ directions.

energy nanocluster. We examined every possible position of nickel atom, which replaced the iron atom, as an analog of random diffused jumps of nickel atom. Also we selected the temperature of $T=300 \mathrm{~K}$ and the distances between atoms of $3.6 \AA$, because the optimal interatomic distances of $\mathrm{Fe}-\mathrm{Ni}-\mathrm{C}$ nanocluster was chosen for simulation in which height of a potential barrier was maximal and fcc nanocluster was the most stable (see Fig. 2) [1012]. In such a system any changes of energy can be made only by changing positions of impurity atoms.

\section{Results and discussion}

Nanocluster energy was calculated based on the location of the carbon atom, taking into account the nickel atom position. We chose two directions of a carbon atom drift to the surface: direction $\langle 022\rangle$ (green arrow) and the way $\langle\overline{1} 11\rangle$ plus $\langle 222\rangle$ (orange arrows) for calculation, which formed a triangle (see Fig. 1). Choice of the way $\langle\overline{1} 11\rangle$ plus $\langle 222\rangle$ was associated with the fact that it was able to pass through the tetrahedral interstice (TIS).
Both directions were energetically favorable for a carbon atom because the cluster energy was almost twice smaller when the carbon atom was on the surface $(L=$ 1.8) compared to its position in the central octahedral interstice $(L=0)$, due to influence of the surface. However, in case when the carbon atom drifted towards $\langle 022\rangle$ direction, the potential barrier $\Delta u(\Delta)$ was higher than two potential barriers $\Delta u_{1}\left(\Delta_{1}\right), \Delta u_{2}\left(\Delta_{2}\right)$ in $\langle\overline{1} 11\rangle$ plus $\langle 222\rangle$ directions (see Fig. 2). We had calculated the energy of an fcc nanocluster of iron at all possible position of a nickel atom, in order to determine its effect on the potential barriers height (see Table I).

TABLE I

Nanocluster energy $\left[\frac{\mathrm{meV}}{\mathrm{atom}}\right]$ at different positions of a carbon and a nickel atom at sequential drift of carbon atom in the direction $\langle\overline{1} 11\rangle$ and $\langle 222\rangle$ to the surface. Cases: (a) $\Delta u_{1} \approx \Delta u_{2}$ (with an accuracy of $5 \%$ ); (b) $\Delta u_{1}>\Delta u_{2}$; (c) $\Delta u_{1}<\Delta u_{2}$, ratios in [\%].

\begin{tabular}{|c|c|c|c|c|c|c|c|}
\hline Ni pos. & Case & $\Delta_{1}$ & $\Delta_{2}$ & $\frac{\Delta_{\min 1,2}}{\Delta_{\max 1,2}}$ & $\Delta$ & $\begin{array}{l}\delta=\Delta- \\
\Delta_{\max 1,2}\end{array}$ & $\frac{\delta}{\Delta}$ \\
\hline $11=14=$ & (a) & 440 & 425 & 3.4 & 534 & 94 & 17.6 \\
\hline$=12$ & & & & & 506 & 66 & 13.0 \\
\hline $2=5 \approx 3=8=$ & (b) & 368 & 343 & 6.8 & 457 & 89 & 19.5 \\
\hline$=6=7$ & & 368 & 343 & 6.8 & 458 & 90 & 19.7 \\
\hline 4 & (b) & 368 & 335 & 9.0 & 427 & 59 & 13.8 \\
\hline $1=$ & (b) & 326 & 216 & 33.7 & 427 & 101 & 13.8 \\
\hline$=9=13$ & & & & & 462 & 136 & 20.3 \\
\hline 10 & (c) & 326 & 362 & -9.9 & 506 & 144 & 28.5 \\
\hline
\end{tabular}

The lowest potential barrier is in the direction of $\langle 022\rangle$ when the nickel atom held positions 1 and 4 , in the case of drift towards $\langle 1 \overline{1} 1\rangle$ plus $\langle 222\rangle$ the potential barrier configuration was more complex. There are three potential barriers' ratios which a carbon atom can overcome using a tetrahedral interstice to reach the surface: (a) $\Delta_{1} \approx \Delta_{2}$; (b) $\Delta_{1}>\Delta_{2}$ and (c) $\Delta_{1}<\Delta_{2}$ (see Table I).

Both of potential barriers with an accuracy of $5 \%$ had an equal height when they corresponded to 11,12 and 14 positions of nickel atom. In these cases, the heights of potential barriers on the way to the surface through tetrahedral interstice were $13-18 \%$ less than in the direction $\langle 022\rangle$. Energy depth of tetrahedral interstices did not exceed $40 \mathrm{meV}$ /atom or $11 \%$ between the maximum and the minimum. This position was the most stable of the three cases, although it was essentially unstable in comparison to the case when a carbon atom occupied the octahedral interstice.

In the second case, which included the majority of positions of a nickel atom $(1 \div 9,13)$, the first barrier was higher than the second potential barrier (see Fig. 3, orange arrows). This created an energy condition for a carbon atom to drift to the surface in order to reduce the nanocluster energy. The energy advantage was from 14 to $20 \%$ in comparison to the direction $\langle 222\rangle$. For the carbon atom, the most energetically favorable was position 1 of a nickel atom.

There was the third case where the height of the second potential barrier of tetrahedral interstice was larger than 


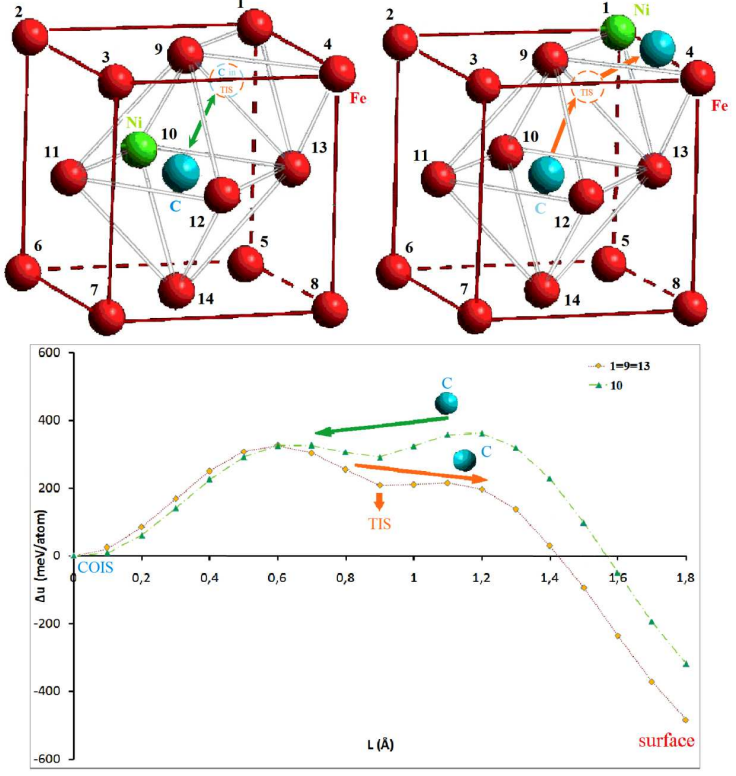

Fig. 3. Scheme of the nickel atom positions that affect the height of potential barriers of the tetrahedral interstice.

the first barrier by $10 \%$. In our opinion, although this height was significantly lower than the potential barrier of an octahedral interstice (by 29\%), the carbon atom drift to the surface through TIS was not energetically favorable, because conditions for returning of a carbon atom to the central octahedral interstice were created (see Fig. 3, green arrows).

\section{Conclusions}

Thus, there are two ways for the carbon atom to drift to the surface of the iron fcc nanocluster: the short direction of $\langle 022\rangle$ with high potential barrier and long direction $\langle\overline{1} 11\rangle$ plus $\langle 222\rangle$, which potential barrier is lower by $13-29 \%$. The carbon atom position in tetrahedral interstice is unstable, so it can be considered as a transitway of the carbon atom to the surface of the nanocluster.
The position of nickel atom affects the height of potential barriers and determines which of the two potential barriers of the tetrahedral interstice is higher. This allows manipulating atoms at the surface of nanocluster.

\section{References}

[1] B.M. Smirnov, Cluster Processes in Gases and Plasmas, Wiley-VCH, Weinheim 2010.

[2] V.V. Sagaradze, V.E. Danilchenko, P. L'Heritier, V.A. Shabashov, Mater. Sci. Eng. 337, 146 (2002).

[3] Z.R. Dai, Sh. Sun, Z.L. Wang, Surf. Sci. 505, 325 (2002).

[4] J. Diao, K. Gall, M.L. Dunn, Nat. Mater. 2, 656 (2003).

[5] L.D. Pachon, G. Rothenberg, Appl. Organomet. Chem. 22, 288 (2008).

[6] E.G. Lewars, Computational Chemistry: Introduction to the Theory and Applications of Molecular and Quantum Mechanics, Springer Science Business Media BV., Berlin 2011.

[7] K.I. Ramachandran, G. Deepa, K. Namboori, Computational Chemistry and Molecular Modelling. Principles and Applications, Springer-Verlag, Heidelberg 2008.

[8] Q. Yang, A.C. To, Comput. Methods Appl. Mech. Eng. 283, 384 (2015).

[9] H.M. Ledbetter, R.P. Reed, J. Phys. Chem. Ref. Data 2, 531 (1973).

[10] T. Halicioğlu, G.M. Pound, Phys. Status Solidi A 30 , 619 (1975).

[11] M. Riech, Nano-Engineering in Science and Technology. An Introduction to the World Nano-Design, World Sci., Singapore 2003.

[12] L.J. Swartzendruber, V.P. Itkin, C.B. Alcock, J. Power Electron. 12, 288 (1991). 